\title{
Drug Induced Sleep Endoscopy (DISE) with Dexmedetomidine (DEX) under Near Infrared Cerebral Spectroscopy (NIRS) in a Pediatric Epileptic Patient
}

\author{
Stefania Franzini, Simona Neri \\ Department of Anesthesia and Pediatric Intensive Care Unit, Fondazione IRCCS Ca' Granda Ospedale Maggiore Policlinico, \\ Milan, Italy.
}

Corresponding Author:

Dr. Stefania Franzini

Email: stefania.franzini@tiscali.it

This is an Open Access article distributed under the terms of the Creative Commons Attribution License (creativecommons.org/ licenses/by/3.0).

Received : September 11, 2018

Accepted : November 7, 2018

Published : November 25, 2018

\begin{abstract}
Background: Presently, no study has been conducted using dexmedetomidine as single agent at maximal dose for drug-induced sleep endoscopy (DISE) under near infrared spectroscopy (NIRS) monitoring. Case Report: Following adenoidectomy for obstructing sleep apnea (OSA), a pediatric patient complained of "absence like" epilepsy. EEG confirmed an epileptiform focus and polysomnography evidenced persistent OSA. To exclude upper airway obstruction, we performed an uneventful DISE under ASA standard monitoring and cerebral NIRS, using DEX as single anesthetic. NIRS evidenced baseline oxygen saturation asymmetry linked to the epileptic focus, which symmetrically slightly decreased during DEX induction, but then remained steady throughout the prolonged examination under DEX as sole anesthetic. Hemodynamic and respiratory profiles did not require any compensatory intervention throughout the examination. Conclusion: According to our finding, DEX even at higher than recommended dose, appears effective and safe as single anesthetic in DISE for OSA in a pediatric epileptic patient.
\end{abstract}

Keywords: Airway Obstruction, Anaesthetics, Electroencephalography, Epilepsy, Oxygen.

\section{Introduction}

Adenotonsillectomy is the first-line treatment of obstructing sleep apnea (OSA) in the pediatric population. However, using polysomnography (PSG) as a metric of cure, upto a third of patients respond [Fig.1] suggesting the presence of fixed or dynamic residual disease. Apneas during sleep are associated with changes in cerebral blood flow to the extent that cerebral auto-regulation may be insufficient to prevent tissue hypoxia, as suggested by MRI spectroscopy [1,2], showing impairment in cerebral metabolism.

The role of dexmedetomidine for druginduced sleep endoscopy (DISE) in refractory OSA is established: it provides a real-time dynamic evaluation of upper airways under direct vision during deep sedation, provided it mimics physiological sleep. However, the use of nonstandardized anesthetic techniques [3] including drugs that possibly decrease the ventilatory drive, induce respiratory depression, cause excessive hypotonia of airways muscles or diaphragm relaxation may jeopardize clinical findings during DISE.

Dexdemetodimine (DEX; Precedex ${ }^{\circledR}$, Hospira, Lake Forest, IL, USA; and Dexdor ${ }^{\circledR}$, Orion Corporation, Espoo, Finland), a short acting, highly potent, highly selective $\alpha 2$-adrenergic receptor agonist, has shown to overcome most of the drawbacks of other anesthetics in DISE. Indeed, it provides anxiolysis, sedation mimicking natural sleep, analgesia, attenuation of the sympathetic hemodynamic response, and minimal respiratory depression even at higher doses and in 
children with OSA [4]. It also preserves airway patency and tone, and obtunds airways reflexes. In addition, DEX has exhibited long-term effects on the developing animal brain, including neuroprotection against anesthetic-induced neuroapoptosis [5], which is particularly important in the pediatric population. DEX for pediatric use has been described for almost a decade in the literature [4] and is commonly employed in DISE, although worldwide there is no approval for administration of this drug to the pediatric population [6].

Many anesthetics used to sedate patients alter oxygen extraction/metabolism by the brain. Near infra-red spectroscopy (NIRS) is a noninvasive, non-pulse oximetry allowing for direct measurement of oxygen in blood in capillaries. Its values mirror surplus of oxygen remaining after the tissues have taken what they require. Regional oxygen saturation declines from baseline as oxygen delivery to an anatomical region decreases or consumption increases, and acts as a trend monitor within individual patients, as each patient serves as his/her own control. A 20\% relative decrease from baseline may have outcome implication. In infants and children, cerebral NIRS consistently detects alterations in oxygenation 15-20 seconds before any change in pulse oximetry [7]. This enhanced detection of early perfusion abnormalities allows for intervention prior to development of hypoxic neuronal injury and potentially prevents postoperative neuro-cognitive sequelae [8].

We therefore decided to perform a DISE in a pediatric epileptic patient under cerebral NIRS monitoring, using DEX as single anesthetic agent during induction and prolonged sedation to assess its safety and effect on cerebral perfusion and oxygenation.

\section{Case Report}

A 5-year old male patient, weighing $19.5 \mathrm{~kg}$, (BMI 20), without co-morbidity and craniofacial abnormality, underwent adenoidectomy for OSA three months previously. At follow-up, he

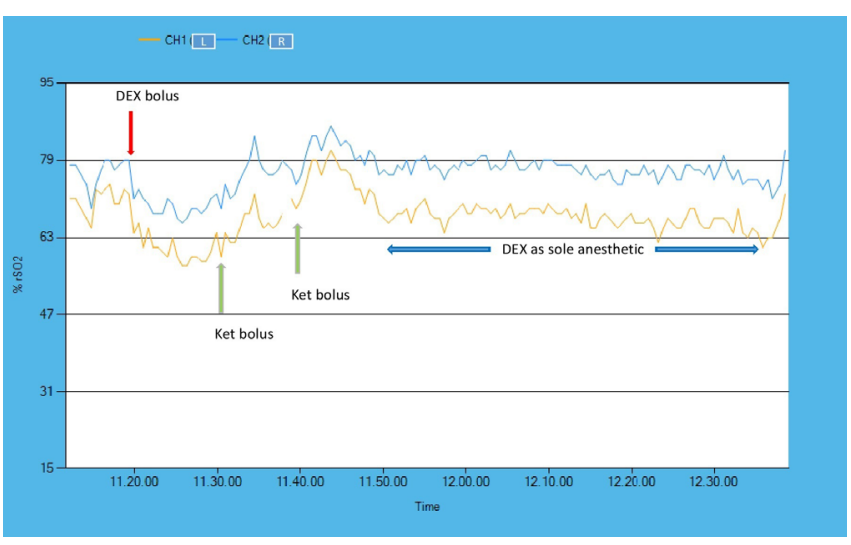

Fig.1: Cerebral NIRS monitoring traces (Ch $1=L$, Ch $2=R)$.

complained of occasional short loss of contact, speech arrest and hypotonia. A PSG showed severe persistent OSA. A brain MRI was normal, while sleep EEG showed rare epileptiform anomalies in the left fronto-temporal area, suggesting abnormal neuronal excitability. To rule out the site(s) of upper airways fixed or dynamic obstruction we performed a DISE before potential additional surgery.

Neither sedative premedication nor atropine was given. In operation theatre the patient was in supine position and spontaneously breathing, with the neck in the natural position taken as asleep. We did not use any topical decongestant/ anesthetic, artificial airway and/or additional airway maneuvers (chin lift, jaw thrust, shoulder roll). Intravenous infusion of normal saline $(250 \mathrm{ml}$ in one hour) compensated for 8 hours fasting and baseline request ( $4 \mathrm{ml} / \mathrm{kg} /$ hour$)$. An intravenous bolus of DEX, $2 \mathrm{mcg} / \mathrm{kg}$, was administered over 10 minutes for induction (starting at 11.20 a.m.) [Fig.1]. To achieve adequate sedation depth, two sedation boluses of ketamine, $1 \mathrm{mg} / \mathrm{kg}$, were administered 10 and 21 at 11.30 and 11.41 am, [Fig.1] minutes after the end of the bolus. The core examination, lasting about 50 minutes, was performed under DEX only, at an infusion rate of 3 $\mathrm{mcg} / \mathrm{kg} / \mathrm{h}$. Monitoring included continuous oxygen saturation, heart rate, electrocardiogram, bilateral cerebral NIRS and q5m non-invasive blood pressure. Recovery from anesthesia took place in the recovery room under standard ASA monitoring. 
No rebound hypertension or tachycardia was observed. We uneventfully completed a full upper airways examination, with operator's satisfaction. A complete transversal collapse with tonsil touch at midline was diagnosed.

\section{Discussion}

In this case, we used a technique that mimics physiological sleep and avoided any airway intervention that might alter the child's physiological and clinical condition [9]. To prevent any drug-induced oxygen desaturation we did not administer a sedative pre-medication. We also avoided atropine to prevent interference with the hemodynamic anticipated effect of DEX (bradycardia or a decrease in resting heart rate). We performed induction using DEX as sole agent. Then we added ketamine at low dose $(1 \mathrm{mg} / \mathrm{kg})$ to deepen sedation. Nevertheless, after the initial nostril instrumentation, we discontinued ketamine and satisfactorily completed the lengthy part of the procedure under continuous infusion of DEX as sole anesthetic agent. As expected, the pharmacological effect of ketamine vanished about 15 minutes after the latest administration. The hemodynamic (in particular MAP values) and respiratory profile, even at high dose DEX, was persistently stable, as already observed [4] and sedation deep. Peripheral oxygen saturation was extremely stable and always above $93 \%$, thus no oxygen supply was needed. Although the risk of an obstruction desaturation may increase during sedation in patients affected by OSA, no apnea occurred, and we clinically noticed only frequent hypopnea's associated with slight increase in respiratory effort. This observation confirms that the use of DEX for sedation seems to reduce the incidence of episodes of oxygen desaturation in children with OSA [4]. In turn, the "protective" effect of DEX in OSA may stem from its effect on upper airways, as it actively maintains airway patency. Under these circumstances, DEX may regrettably conceal potential dynamic changes of upper airways leading to apnea in OSA. Overall, the dose of DEX we used, although higher than recommended, fulfills the commonly employed dosing regimens for intra-operative airway procedures [4], including the modality of administration.

To the best of our knowledge, this is the first report of DISE performed under NIRS monitoring in a child. During the DEX initial bolus infusion (from 11.20 to 11.30 a.m), [Fig.1] NIRS promptly evidenced a baseline asymmetric brain oxygen extraction (L>R) [Fig.1], which persisted throughout the examination, suggesting focal metabolic brain impairment. This focus of altered brain activity matched the sleep EEG, which showed epileptiform anomalies in the left frontotemporal area. Our observation confirms that DEX preserves epileptiform activity in children with seizure disorders [10]. In addition, at induction, under sole DEX bolus infusion, a symmetrical decrease, although clinically not significant, of brain oxygen saturation ensued, confirming previous observations [11]. However, in that earlier experience pre-medication with midazolam, at a non-specified regimen, might have altered the actual effect of DEX on brain oxygen saturation. This was not the case in our study. In the absence of any interfering drug or maneuver, in a patient with stable hemodynamic and respiratory profile, brain oxygen desaturation was probably due to cerebral arterial vasoconstriction caused by the $\alpha-2$ effect of DEX on alfa-adrenoceptors located on cerebral pia [12]. At the same time, pulse oximetry failed to detect any anomaly in peripheral oxygen saturation, confirming that brain oxygen desaturation may occur in children inspite of steady pulse oximetry.

In order to ease upper airway instrumentation, we deepened sedation with ketamine. This did not alter the observed baseline asymmetry of cerebral oxygenation and caused a predictable symmetrical reduction of oxygen extraction (Fig.1, min 11.30, and 11.41 a.m.), that returned to baseline values as ketamine was metabolized ( 11.50, Fig.1). Yet, during the residual procedure performed under DEX as sole anesthetic 
drug at higher dose, NIRS showed a steady symmetrical brain oxygen saturation matching the baseline (11.50-12.40 a.m., Fig.1).

\section{Conclusion}

Our experience suggests that a complete satisfactory DISE can be safely attained in a pediatric patient on high dose DEX as single anesthetic agent, with stable hemodynamic and respiratory profiles, without significant alteration of the baseline brain tissue oxygen extraction, in particular at higher doses and during extended sedation. DEX allows recognizing areas of impaired brain metabolism. Further studies are needed to confirm this observation.

Contributors: SF and SN designed and conducted the study. SF collected and analyzed the data and wrote the manuscript. SF and SN critically revised for important intellectual contents. SF will act as guarantor of study. Both authors have read and approved the final manuscript.

Funding: None; Competing interests: None stated.

\section{References}

1. Friedman $\mathbf{M}$, Wilson $\mathrm{M}$, Lin $\mathrm{H}$, Chang H. Updated systematic review of tonsillectomy and adenoidectomy for treatment of pediatric obstructive sleep apnea/ hypopnea syndrome. Otolaryngol Head Neck Surg. 2009;140:800-808.

2. Olopade CO, Mensah E, Gupta R, Huo D, Picchietti DL, Gratton $\mathrm{R}$, et al. Noninvasive determination of brain tissue oxygenation during sleep apnea: a near-infrared spectroscopic approach. Sleep. 2007;30:1747-1755.

3. Friedman NR, Parikh SR, Ishman SL, Ruiz AG, El-Hakim H, Ulualp SO, et al. The current state of pediatric drug-induced sleep endoscopy. Laryngoscope. 2017;127:266-272.

4. Mahmoud M, Mason KP. Dexmedetomidine: review, update, and future considerations of pediatric perioperative and periprocedural applications and limitations. Br J Anaesth. 2015;115:171-182.

5. Sanders RD, Sun P, Patel S, Li M, Maze M, Ma D. Dexmedetomidine provides cortical neuroprotection: impact on anaesthetic-induced neuroapoptosis in the rat developing brain. Acta Anaesthesiol Scand. 2010;54:710-716.

6. Mason KP., Lerman J. Dexmedetomidine in children: current knowledge and future applications. Anesth Analg. 2011;113:1129-1143.

7. Tobias JD. Cerebral oximetry Monitoring with near infrared spectroscopy detects alterations in oxygenation before pulse oximetry. $\mathrm{J}$ Intensive Care Med. 2008;23:384-388.

8. Giliberti P, Mondì V, Conforti A, Haywood Lombardi M, Sgrò S, Bozza $\mathrm{P}$, et al . Near infrared spectroscopy in newborns with surgical disease. J Matern Fetal Neonatal Med. 2011; 24:56-58.

9. Chatterjee D, Friedman N, Shott S, Mahmoud M. Anesthetic dilemmas for dynamic evaluation of the pediatric upper airway. Semin Cardiothorac Vasc Anesth. 2014;18:371-378.

10. Mason KP, O'Mahony E, Zurakowski D, Libenson MH. Effects of dexmedetomidine sedation on the EEG in children. Paediatr Anaesth. 2009;19:1175-1183.

11. Cetin M, Birbicer H, Hallioglu O, Orekeci G. Comparative study between the effects of dexmedetomidine and propofol on cerebral oxygenation during sedation at pediatric cardiac catheterization. Ann Card Anaesth. 2016;19:20-24.

12. Everett LL, Van Rooyen IF, Warner MH, Shurtleff HA, Saneto Do RP, Ojemann JG. Use of dexmedetomidine in awake craniotomy in adolescents: report of two cases. Pediatr Anesth. 2006;16:338-342. 